\title{
An Improved Specific Harmonic Detection Method Based on Adaptive Cancellation Principle
}

\author{
Lei Wang ${ }^{1 *}$, Sun Pan $^{1}$, Sun Jun ${ }^{1}$, Liang Yan ${ }^{1}$, Xie Hai Lang ${ }^{1}$, Xiong Sheng ${ }^{1}$ \\ ${ }^{1}$ School of Electrical Engineering, Naval University of Engineering, Wuhan, Hubei, 430033, China
}

\begin{abstract}
When the active power filter (APF) and the tunable filter are used for specific harmonic suppression, the accurate single harmonic current detection module is one of the key links to ensure its harmonic suppression effect. An improved single harmonic detection method based on the principle of adaptive cancellation has been proposed, which does not have some of the problems of traditional algorithms, for instance the feedback error is not accurate enough, and the contradiction between tracking speed and steady-state accuracy. This method can accurately and quickly detect the harmonic amplitude and phase angle, and can be flexibly applied to the harmonic detection of three-phase and single-phase systems. Through theoretical analysis and MATLAB simulation, it is proved that the method can guarantee a higher steady-state accuracy and a faster tracking speed.
\end{abstract}

\section{Introduction}

In recent years, as more and more nonlinear loads are put into use at the end of the power grid, the harmonic current generated by them seriously affects the power quality of the power grid [1]. The existence of harmonic current can easily lead to the malfunction of all kinds of protection devices, so that some precision instruments can't work normally. It may even cause series/parallel resonance between inductive impedance and capacitive impedance in the power network, resulting in the collapse of the power network. Existing main harmonic suppression devices are active power filter (APF), tunable filter and passive filter. Literature [2][3] respectively elaborated the working principle of APF and tunable filter in detail. Combined with the working principle of APF and tunable filter for specific harmonic suppression, it can be seen that a fast and accurate single harmonic detection method is one of the key steps to ensure that these two kinds of harmonic control devices have good harmonic suppression effect. Therefore, it is very important to study a fast and accurate specific harmonic detection method which can be flexibly applied to single phase and three phase systems.

The main specific harmonic detection methods include the harmonic detection method based on the fast Fourier transform, the harmonic detection method based on the instantaneous reactive power theory [4-5], the harmonic detection method based on the artificial neural network [6], and the harmonic detection method based on the adaptive cancellation principle. The harmonic detection method based on fast Fourier transform is only suitable for the detection of stable signal, and is not suitable for the occasion of the load changing frequently. All harmonic detection methods based on instantaneous reactive power theory must adopt low-pass filter, which will cause large detection error and delay, and this method cannot be flexibly applied to single-phase and three-phase system harmonic detection. The harmonic detection method based on artificial neural network involves the training of network parameters and the algorithm is more complex. The traditional harmonic detection method based on the principle of adaptive cancellation is difficult to give consideration to the tracking speed and steady precision. This paper mainly studies based on the principle of adaptive cancellation is harmonic test, aiming at the problems existing in the traditional adaptive single harmonic test, an improved adaptive single harmonic method, this method does not need complex variable step iterative formula, only need to select suitable step length parameters, can be to gain fast tracking speed, and high steady precision, and finally the superiority of the improved algorithm is verified by simulation.

\section{Overview of traditional harmonic detection methods based on adaptive cancellation principle}

In Literature [7-9], the adaptive cancellation technology is introduced in detail, and its principle block diagram is shown in Figure. 1. 


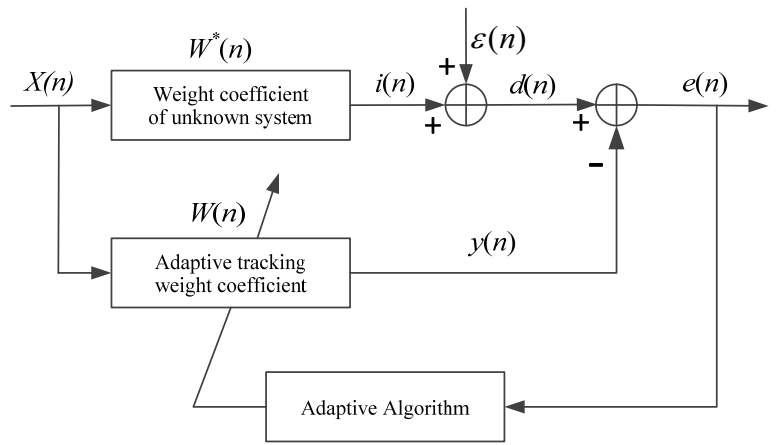

Figure 1. Schematic diagram of adaptive cancellation technology

In Figure. $1, n$ is the current sampling point, the input signal $X(n)=\left[x_{1}(n), x_{2}(n), \ldots, x_{M}(n)\right]^{T}, \quad M$ is the unknown system's dimension. $W^{*}(n)$ is the unknown system weight coefficient matrix, $W(n)$ is the tracking weight coefficient matrix of the adaptive filter, $\varepsilon(n)$ is the noise signal, $i(n)$ is the target signal for tracking, $X(n)$ is related to $i(n)$, but not to $\varepsilon(n)$. Through the iterative process, the adaptive filter makes the output signal $y(n)$ approximate $i(n), W(n)$ approximate $W^{*}(n)$, which $e(n)$ is the tracking error. Each signal in Figure 1 satisfies the following relationship:

$$
\begin{gathered}
W^{*}(n)=\left[w_{1}^{*}(n), w_{2}^{*}(n), \ldots, w_{M-1}^{*}(n), w_{M}^{*}(n)\right]^{T} \\
W(n)=\left[w_{1}(n), w_{2}(n), \ldots, w_{M-1}(n), w_{M}(n)\right]^{T} \\
i(n)=X^{T}(n) W^{*}(n) \\
y(n)=X^{T}(n) W(n) \\
e(n)=d(n)-y(n)
\end{gathered}
$$

According to the literature [10-13], $W(n)$ is updated iteratively with the goal of minimizing $J=|e(n)|^{2}$, and the iterative update formula of $W(n)$ is obtained as follows:

$$
W(n+1)=W(n)+\mu e(n) X(n)
$$

Where, $\mu$ is the step size factor, which is the key factor to balance the convergence speed and steady state error of the algorithm. The larger the $\mu$ is, the faster the convergence speed will be, but it will lead to larger steady-state error and even lead to divergence of the algorithm. The smaller the $\mu$ is, the smaller the steady- state error will be, but the convergence speed will be reduced.

In summary, in Figure $1, d(n)$ is regarded as the load current containing harmonics, $i(n)$ is regarded as the target harmonic to be detected, and $\varepsilon(n)$ is the difference between the load current and the target harmonic. In this case, it can be seen from the orthogonality of the trigonometric function that $i(n)$ and $\varepsilon(n)$ are not related. Therefore, according to the working principle of the adaptive cancellation principle, for different targets to be detected, as long as an input signal $X(n)$ related to $i(n)$ is constructed, the adaptive cancellation principle can be well applied to the harmonics of the power system. It should be noted that for different target sub-harmonics, the input signal $X(n)$ is different, and the detection model and the implementation steps of the algorithm are also different.

According to the desired harmonic suppression effect, the detection target signal can be mainly divided into two cases: that is, the target harmonic is the active component of the fundamental current or the $\mathrm{m}$-th harmonic. Literature [14] elaborates and improves the algorithm for the target signal being the active component of the fundamental current. The focus of this paper is to improve the traditional algorithm for the target signal being any $m$-th harmonic.

\section{The detection model when the target signal is $\boldsymbol{m}$-th harmonic component}

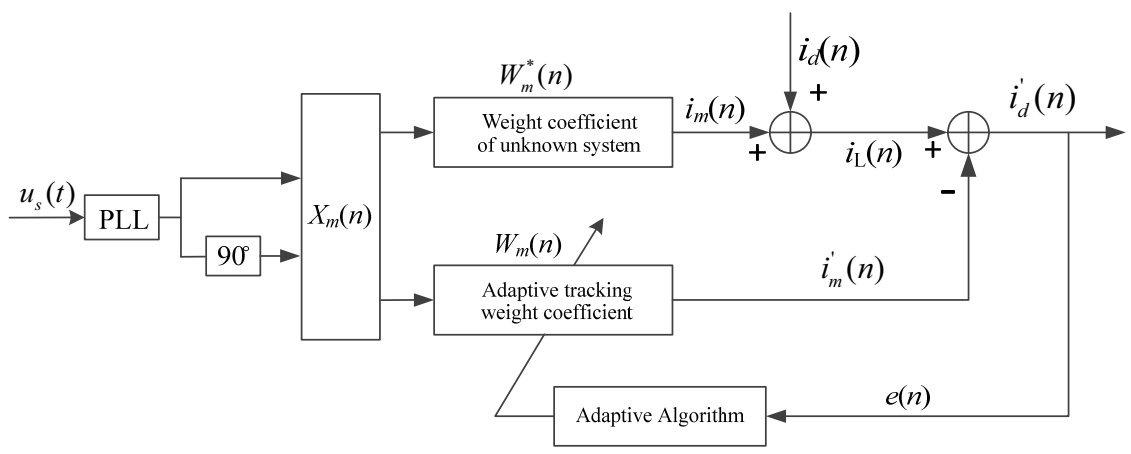

Figure 2. Schematic block diagram when the detection target is $\mathrm{m}$ harmonic 
When the target signal is $m$-th harmonic ( $m=1$ represents the fundamental wave), its detection model is shown in Figure 2. At this point, it is assumed that the power grid voltage signal is: $u_{s}(t)=U_{s} \sin \omega t$, The input signal $X_{m}(n)=\left[\sin m \omega n T_{s}, \cos m \omega n T_{s}\right]^{T}$ is obtained by phase-locked loop and frequency doubling. In the figure, $i_{m}(n)$ is the $m$-th harmonic to be measured. $i_{m}^{\prime}(n)$ is the tracking signal output by the adaptive filter. The

$$
\begin{aligned}
i_{L}(n) & =i_{m}(n)+i_{d}(n)=I_{m} \sin \left(m \omega n T_{s}+\varphi_{m}\right)+\sum_{\substack{k=1 \\
k \neq m}}^{K} I_{k} \sin \left(k \omega n T_{s}+\varphi_{k}\right) \\
& =\underbrace{I_{m} \cos \varphi_{m} \sin m \omega n T_{s}+I_{m} \sin \varphi_{m} \cos m \omega n T_{s}}_{i_{m}(n)}+\underbrace{\sum_{\substack{k=1 \\
k \neq m}}^{K} I_{k} \sin \left(k \omega n T_{s}+\varphi_{k}\right)}_{i_{d}(n)}
\end{aligned}
$$

According to Equation (7), the weight of the adaptive filter is $W_{m}(n)=\left[w_{m 1}(n), w_{m 2}(n)\right]^{T}$, the system weight coefficient matrix $W_{m}^{*}(n)=\left[w_{m 1}^{*}(n), w_{m 2}^{*}(n)\right]^{T}=\left[I_{m} \cos \varphi_{m}, I_{m} \sin \varphi_{m}\right]^{T}$.

Then the iteration formula of weight update is as follows:

$$
W_{m}(n+1)=W_{m}(n)+\mu e(n) X_{m}(n)
$$

That is,

$$
\begin{gathered}
w_{m 1}(n+1)=w_{m 1}(n)+\mu_{m 1} e(n) x_{m 1}(n) \\
w_{m 2}(n+1)=w_{m 2}(n)+\mu_{m 2} e(n) x_{m 2}(n)
\end{gathered}
$$

adaptive filter makes $W_{m}(n)$ approach the system weight coefficient $W_{m}^{*}(n)$ by iteratively updating the weight coefficient, and makes $i_{m}^{\prime}(n)$ approximate $i_{m}(n)$, $i_{d}^{\prime}(n)$ approximate $i_{d}(n)$.

At this point, the load current is shown in Equation

Then the amplitude and phase of the tracking signal $i_{m}^{\prime}(n)$ are as follows:

$$
\begin{aligned}
I_{m}^{\prime}(n) & =\sqrt{w_{m 1}^{2}(n)+w_{m 2}^{2}(n)} \\
\varphi_{m}^{\prime}(n) & =\arctan \frac{w_{m 2}(n)}{w_{m 1}(n)}
\end{aligned}
$$

\section{Algorithm's improvement}

According to Figure. 2, the traditional error feedback signal is shown in Equation (13):

$$
\begin{aligned}
e(n) & =i_{L}(n)-i_{m}^{\prime}(n)=X_{m}^{T}(n)\left[W_{m}^{*}(n)-W_{m}(n)\right]+i_{d}(n) \\
& =x_{m 1}(n)\left[w_{m 1}^{*}(n)-w_{m 1}(n)\right]+x_{m 2}(n)\left[w_{m 2}^{*}(n)-w_{m 2}(n)\right]+i_{d}(n) \\
& =\underbrace{\left[w_{m 1}^{*}(n)-w_{m 1}(n)\right]}_{e_{r m 1}(n)} \sin m \omega n T+\underbrace{\left[w_{m 2}^{*}(n)-w_{m 2}(n)\right]}_{e_{r m 2}(n)} \cos m \omega n T_{s}+i_{d}(n)
\end{aligned}
$$

Through observation and analysis of Equation (13), it can be found that, $e(n)$ cannot directly reflect the tracking situation of $\mathrm{m}$-th harmonics, while $e_{r m 1}(n)$, $e_{r m 2}(n)$ in Equation (13) are the error signals that can directly reflect the adaptive filter weight coefficient tracking the unknown system weight coefficient. Therefore, the update of $w_{m 1}(n)$ and $w_{m 2}(n)$ can be controlled by using $e_{r m 1}(n)$ and $e_{r m 2}(n)$ as the feedback of adaptive system, so that the tracking signal

$$
\begin{aligned}
e(n) \sin \left(m \omega n T_{s}\right) & =\left[e_{r m 1}(n) \sin \left(m \omega n T_{s}\right)+e_{r m 2}(n) \cos \left(m \omega n T_{s}\right)+i_{d}(n)\right] \sin \left(m \omega n T_{s}\right) \\
& =\frac{e_{r m 1}(n)}{2}+\xi_{m 1}
\end{aligned}
$$

Multiply both sides of Equation (13) by $\cos \left(m \omega n T_{s}\right)$, after simplification and arrangement, Equation (15) can be obtained: 


$$
\begin{aligned}
e(n) \cos \left(m \omega n T_{s}\right) & =\left[e_{r m 1}(n) \sin \left(m \omega n T_{s}\right)+e_{r m 2}(n) \cos \left(m \omega n T_{s}\right)+i_{d}(n)\right] \cos \left(m \omega n T_{s}\right) \\
& =\frac{e_{r m 2}(n)}{2}+\xi_{m 2}
\end{aligned}
$$

Equations (11) and (15) are respectively integrated, and the integral range is half of the grid voltage cycle, that is $T / 2$. It has been proved that the result of simplification of Equations (14) and (15), $e_{r m 1}(n)$ and $e_{r m 2}(n)$ can be extracted, as shown in Equations (16) integration of $\xi_{m 1}$ and $\xi_{m 2}$ in the range of T/2 is zero. and (17).

Therefore, after the above operation processing and

$$
\begin{aligned}
& e_{r m 1}(n)=\frac{4}{N} \sum_{i=n-N / 2+1}^{n} e(i) \sin \left(m \omega i T_{s}\right)+\frac{2}{N} \sum_{i=n-N / 2+1}^{n-1} w_{m 1}(i)+\frac{2-N}{N} w_{m 1}(n) \\
& e_{r m 2}(n)=\frac{4}{N} \sum_{i=n-N / 2+1}^{n} e(i) \cos \left(m \omega i T_{s}\right)+\frac{2}{N} \sum_{i=n-N / 2+1}^{n-1} w_{m 2}(i)+\frac{2-N}{N} w_{m 2}(n)
\end{aligned}
$$

The extracted en 1 and en2 are taken as error feedback quantity to obtain a new weight update iterative formula as follows:

$$
\begin{gathered}
w_{m 1}(n+1)=w_{m 1}(n)+\mu_{m 1} e_{r m 1}(n) \\
w_{m 2}(n+1)=w_{m 2}(n)+\mu_{m 2} e_{r m 2}(n)
\end{gathered}
$$

According to the above theoretical analysis, the weight of the adaptive filter can be tracked to the system weight coefficient after half a cycle of iterative update if

\begin{tabular}{|c|c|}
\hline & Improved algorithm for tracking $i_{\mathrm{m}}(t)$ \\
\hline Initialize & $\begin{array}{c}W_{m}(0)=\left[w_{m 1}(n), w_{m 2}(n)\right]^{T}=[0,0]^{T} \\
\mu_{m 1}(0)=0, \mu_{m 2}(n)=0\end{array}$ \\
\hline Feedback error & $e_{r m 1}(n), e_{r m 2}(n)$ \\
\hline $\begin{array}{c}\text { Algorithm } \\
\text { implementation } \\
\text { process }\end{array}$ & $\begin{array}{l}\begin{array}{l}\text { For } n=0,1,2, \ldots \\
y(n)=W^{T}(n) X(n) \\
e(n)=i_{L}(n)-y(n)\end{array} \\
\begin{aligned} & e_{r m 1}(n)=\frac{4}{N} \sum_{i=n-N / 2+1}^{n} e(i) \sin \left(m \omega i T_{s}\right)+\frac{2}{N} \sum_{i=n-N / 2+1}^{n-1} w_{m 1}(i)+\frac{2-N}{N} w_{m 1}(n) \\
& e_{r m 2}(n)=\frac{4}{N} \sum_{i=n-N / 2+1}^{n} e(i) \cos \left(m \omega i T_{s}\right)+\frac{2}{N} \sum_{i=n-N / 2+1}^{n-1} w_{m 2}(i)+\frac{2-N}{N} w_{m 2}(n) \\
& w_{m 1}(n+1)=w_{m 1}(n)+\mu_{m 1} e_{r m 1}(n) \\
& w_{m 2}(n+1)=w_{m 2}(n)+\mu_{m 2} e_{r m 2}(n)\end{aligned} \\
\text { end } \quad\end{array}$ \\
\hline
\end{tabular}
the appropriate step size is selected.

According to the foregoing, the algorithm flow of the improved harmonic detection method is shown in Table 1 .

Table 1. Algorithm flow of improved harmonic detection method

algorithm was simulated on the MATLAB platform, and

\section{The simulation analysis}

In order to more clearly observe the tracking of the target sub-harmonics by the adaptive filter, the detection

$$
i_{L}(t)=I_{1} \sin (\omega t+80)-I_{5} \sin (5 \omega t+45)+1.5 \sin (\omega t+36)+0.9 \sin (11 \omega t+30)
$$

Wherein, $\omega$ is the angular frequency, $\omega=2 \pi f$, $f=50 \mathrm{~Hz}, I_{1}$ is the fundamental current amplitude, and $I_{5}$ is the fifth harmonic amplitude.

The detection target harmonic is the fifth harmonic. According to the foregoing, the reference input signal at this time is:

$$
X(n)=[\sin (5 \omega t), \cos (5 \omega t)]
$$

\subsection{When the load current is a stationary signal}

When the load current $I_{1}=10$ and $I_{5}=2$, the step size $\mu_{m 1}=\mu_{m 2}=0.08$ was selected. The traditional algorithm and the improved algorithm proposed in this paper were respectively used to track the fifth harmonic. The simulation results were shown in Figure 3 and Figure 4. Figure 3 shows the load current waveform at this time. Figure 4(a) shows the tracking of the fifth 
harmonic by the improved algorithm proposed in this paper, and Figure4(b) shows the tracking of the traditional algorithm. It can be seen that the improved algorithm has obvious advantages over the traditional algorithm in tracking the target subharmonic.

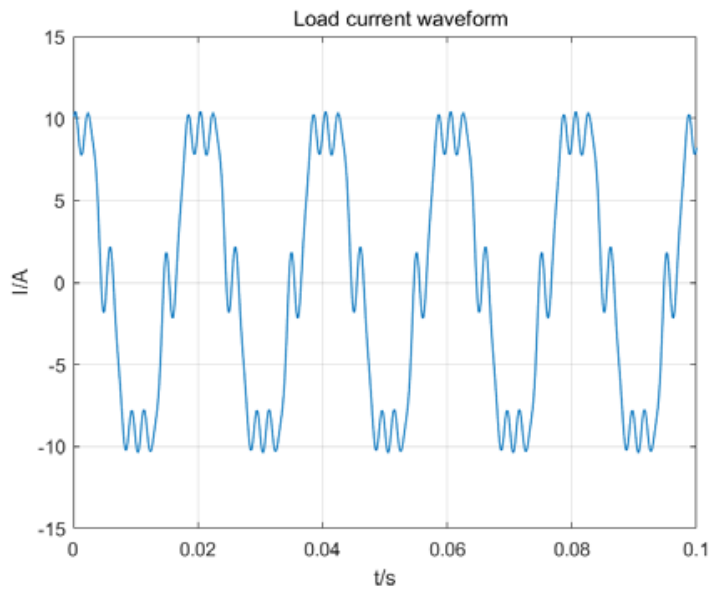

Figure. 3 Waveform of load current

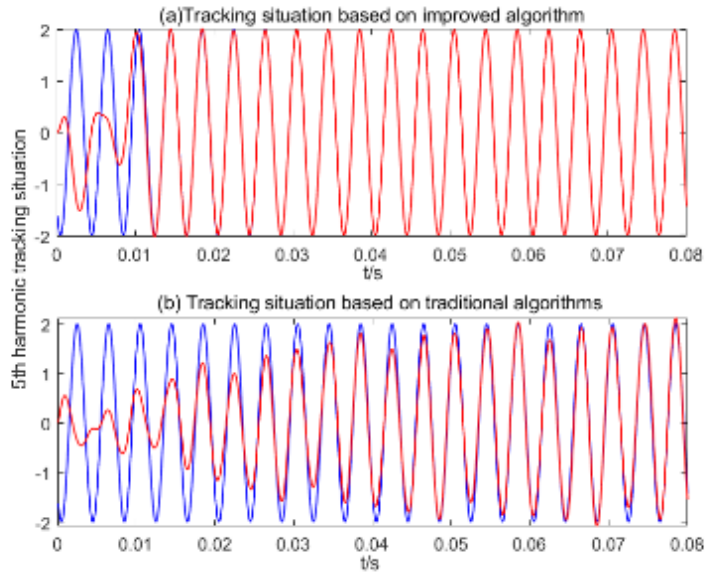

Figure 4. Comparison diagram of fifth harmonic detection

\subsection{When the load changes}

In the simulation, the amplitude of the fundamental wave component and the fifth harmonic component of the load current mutated at $0.04 \mathrm{~s}$ in the simulation. $I_{1}$ mutates from $10 \mathrm{~A}$ to $15 \mathrm{~A}, I_{5}$ mutates from $2 \mathrm{~A}$ to $3 \mathrm{~A}$, and the simulation results are shown in Figure 5, Figure 6 and Figure 7. Figure 5 shows the tracking of the fifth harmonic in the abrupt load current when the improved algorithm is used. Figure 6 shows the amplitude and phase angle of the corresponding tracking signal. Figure 7 shows the change of the corresponding tracking error. It can be seen that the improvement algorithm can react quickly after a sudden change in the load current, tracking the target harmonic in about half a cycle, and then after a small fluctuation of half a power grid cycle, it can accurately track the amplitude and phase angle of the target sub-harmonic. Therefore, the improved algorithm can be applied to occasions where the load is frequently switched to.

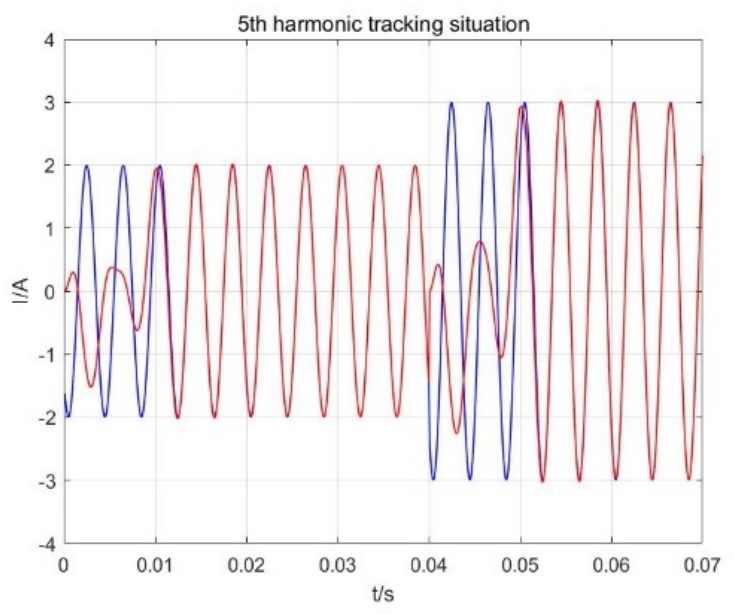

Figure 5. Tracking the fifth harmonic when the load changes suddenly
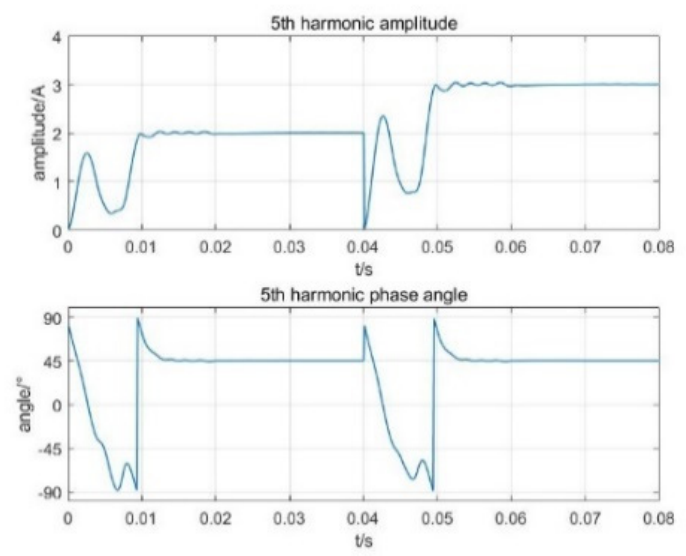

Figure 6. Tracking the fifth harmonic amplitude and phase angle when the load changes suddenly

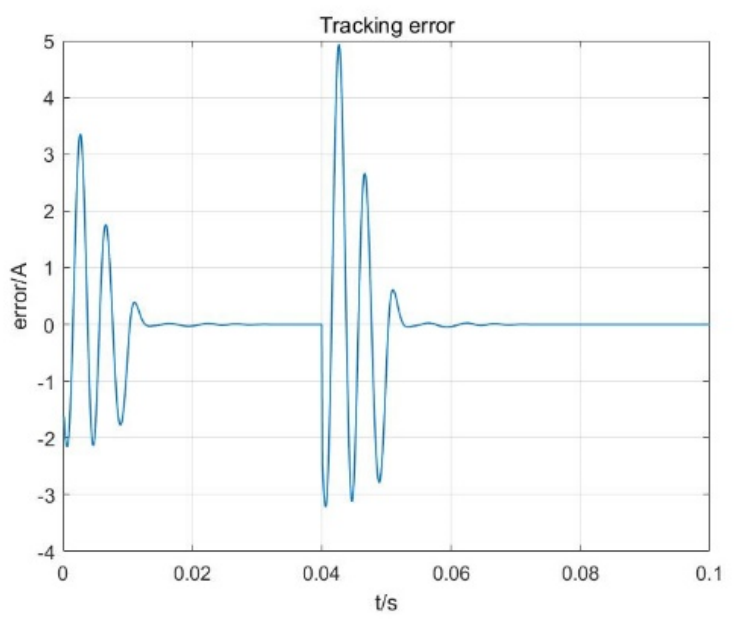

Figure 7. Error change of tracking the fifth harmonic when the load changes suddenly

\section{Conclusion}

Aiming at the problems of inaccurate feedback error and too complicated step-size iteration update formula in the traditional adaptive harmonic detection method, this paper proposes an improved specific harmonic detection 
method based on the principle of adaptive cancellation. This method combines the orthogonality of the trigonometric function to extract the accurate tracking error, replaces it with the error feedback in the traditional algorithm, and then realizes the update of the tracking filter weight by selecting the appropriate step parameter. For a three-phase system, as long as the single-phase detection method discussed above is applied to the threephase current detection, the single-harmonic detection of the three-phase system can be realized. Therefore, the method can also be flexibly applied to single-phase and three-phase systems. The simulation proves that the algorithm has better tracking effect than the traditional adaptive single harmonic detection method, and it has good adaptability to sudden current signals.

\section{Acknowledgments}

This research was funded by National Natural Science Foundation of China (No. 5180719 and No. 52007195) and Group Project in Hubei Province Natural Science Foundation of Innovation (No. 2018CFA008).

\section{References}

1. Zhang Yu xiao, Dai Ke, Chen Xin wen, Zhang San hong, Kang Yong. A new method of SAPF harmonic suppression and resonance damping for detecting current including capacitive current $[\mathrm{J}]$. Transactions of the Chinese Society of Electrical Engineering,2017,32(S1):58-67.

2. Zhang Jian zhong, Geng Zhi, Xu Shuai, Chen Hao. An improved adaptive harmonic detection algorithm for active power filter[J]. Transactions of the Chinese Society of Electrical Engineering,2019,34(20):4323-4333.

3. Yu Mingtao, Chen Jian ye, Wang Zanji. Singletuned filter based on controllable reactor[J]. Power Automation Equipment, 2007, 027(001):39-41.

4. Zhang Chao, Yang Geng, Du Jihong. A new algorithm for arbitrary harmonic current detection of active power filter[J]. Journal of Electrical Machines and Control, 2002, 006(003):252-255.

5. Yin Chun jie, Wang Fang, Shi Lei, Zhang Cheng hui, Zhang Mei. Active power filter compensation current detection based on instantaneous voltage vector orientation[J].Transactions of the Chinese Society of Electrical Engineering, 2017,32(07):112118.

6. Ma Li xin, Wang Xiao dan, Wang Yue xiao, et al. Research on Intelligent Detection Method of APF Specific Subharmonics[J]. Control Engineering, 2013, 020(002):352-356.

7. Wang Shuo, Kang Jin song. A method of extracting and suppressing current harmonics of permanent magnet synchronous motor based on adaptive linear neural network algorithm[J]. Transactions of the Chinese Society of Electrical Engineering, 2019,34(04):654-663.
8. Zhao $\mathrm{H}, \mathrm{Yu} \mathrm{Y}$, Gao S, et al. A new normalized LMAT algorithm and its performance analysis[J]. Signal Processing, 2014, 105:399-409.

9. Mayyas, Khaled. A variable step-size selective partial update LMS algorithm[J]. Digital Signal Processing, 2013, 23(1):75-85.

10. Bismor D. LMS Algorithm Step Size Adjustment for Fast Convergence[J]. Archives of Acoustics, 2012, 37(1):31-40.

11. Song Zhi xiong, Yu Yi, Zhao Hai quan. Variable step size adaptive harmonic current detection algorithm based on skip-tongue function[J]. Automation of Electric Power Systems, 2013, 37(22):54-59.

12. Liu Chuan lin, Liu Kai pei. LMS-based accelerated convergence step selection principle and adaptive three-phase power grid harmonic detection[J]. Power Automation Equipment, 2013,33(04):77-81.

13. Zhou Tian hao, Chen Lei, Zhu Changsheng, Li Peng fei. Unbalance compensation of magnetic levitation high-speed motor based on adaptive variable step size least mean square algorithm[J]. Transactions of the Chinese Society of Electrical Engineering, 2020,35(09):1900-1911.

14. Wang Hong cheng, Wang Lei, Shen Xia, Yang Xin rong, Wang Zheng. Harmonic detection method based on improved gain type adaptive LMS algorithm $[\mathrm{J}]$. Power System Protection and Control, 2016,44(05):42-46. 